\title{
PROMOÇÃO dO PAPEL PARENTAL NOS COMPORTAMENTOS DE SONO DE CRIANÇAS ATÉ AOS CINCO ANOS DE IDADE
}

\author{
Dulce Maria Pereira Garcia Galvão \\ (Pós Doutoramento em Enfermagem, Professora Coordenadora na Escola Superior de Enfermagem de \\ Coimbra - ESEnfC - Coimbra, Portugal.) \\ dgalvao@esenfc.pt \\ Ana Laura Monteiro Carpenter \\ (Licenciatura em Enfermagem, Escola Superior de Enfermagem de Coimbra - ESEnfC - Coimbra, Portugal.) \\ Paula Cristina Alves Castro \\ (Licenciatura em Enfermagem, Escola Superior de Enfermagem de Coimbra - ESEnfC - Coimbra, Portugal.) \\ Telma Isabel Monteiro Almeida \\ (Licenciatura em Enfermagem, Escola Superior de Enfermagem de Coimbra - ESEnfC - Coimbra, Portugal.) \\ Escola Superior de Enfermagem de Coimbra. Avenida Bissaya Barreto, apartado 55. CEP 3001-901. Coimbra, \\ Portugal.
}

\section{RESUMO}

Antecedentes: 0 sono assume papel importante nos primeiros anos de vida ao promover 0 crescimento e desenvolvimento da criança. A insuficiência e baixa qualidade do sono nas crianças tem vindo a constituir problema de saúde pública. Um total de 25 a $50 \%$ das crianças até aos cinco anos de idade apresentam dificuldades relacionadas com 0 sono. Objetivos: Conhecer as intervenções de enfermagem desenvolvidas pelos enfermeiros que visem o exercício adequado do papel parental no lidar com o comportamento dos filhos no momento de dormir. Participantes/Métodos: Revisão Integrativa da Literatura, para responder à questão "Como ajudam os enfermeiros os pais de crianças até aos cinco anos de idade a lidar com o comportamento dos filhos no momento de dormir?" Pesquisa bibliográfica nas bases de dados social science, nursing and heath professions, neuroscience, medicine and dentistry decision science, B-on, Psychology and Behavioral Sciences Collection, Medline (with full-text), CINAHL Plus (with full-text), MedicLatina and ERIC, selecionando estudos de 2012 a Maio/2017 em português, inglês, francês e espanhol utilizando-se "parents"; "nurse"; "children's sleep"; "Sleep habits"; "sleep"; "children" e "recommendation" e seus correspondentes em português. Incluíram-se oito artigos. Resultados: Pais com maior conhecimento sobre higiene e padrões de sono dos filhos, adotam melhores estratégias, mais adequadas às suas crianças e que visam a promoção de comportamentos favorecedores do sono. Existem algumas dis- 
paridades sobre Bed Sharing e sestas em contexto de infantário. Os enfermeiros, de uma forma geral, têm pouca formação sobre o sono da criança, o que dificulta a sua ação. Conclusões: Embora a maioria dos pais estejam sensíveis a desenvolver estratégias adequadas às necessidades de sono do filho, os enfermeiros devem estar despertos para a importância da capacitação dos pais sobre 0 sono dos filhos. Tem de ser feito um maior investimento na vertente dos ensinos e promoção de boas práticas antes do sono.

Palavras-Chave: enfermeiro; pais; higiene do sono; criança

\section{ABSTRACT}

Promoting parental role in the child's sleep behaviors up to five years old.

Introduction: The sleep plays an important role in the first years of life to promote the growth and development of children. The insufficiency and low quality of sleep in children has been a public health problem. A total of 25 to $50 \%$ of children up to five years old present difficulties related sleep. Objectives: To know the nursing interventions developed by the nurses aimed at the adequate exercise of parental role in dealing with children's behavior at bedtime. Methods: Integrative Literature Review, to answer the question "How nurses help the parents of children up to five years old to deal with their children's behavior at bedtime?" Bibliographic search in databases social science, nursing and health professions, neuroscience, medicine and dentistry decision science, B-on, Psychology and Behavioral Sciences Collection, Medline (with full-text), CINAHL Plus (with fulltext), MedicLatina and ERIC, selecting studies from 2012 to May/2017 in Portuguese, English, French and Spanish using the descriptors "parents", "nurse", "children's sleep", "Sleep habits", "sleep", "children" e "recommendation" and their correspondents in Portuguese. They included eight articles. Results: Parents with greater knowledge about children's hygiene and sleep patterns adopt better strategies, more appropriate to their children and that aim to promote sleep-favoring behaviors. There are some differences about bed sharing and naps in the context of Nurseries. The nurses, in general, have little education about the child's sleep, which makes their action difficult. Conclusion: Although most of parents are sensitive to developing strategies appropriate to their child's sleep needs, the nurses should be awake to the importance of parent empowerment about children's sleep. There must be a greater investment in the teaching and promotion of good practices before sleep.

Key-Words: nurses; parents; sleep hygiene; child

\section{INTRODUÇÃO}

0 sono, necessidade humana fundamental de todos os indivíduos, assume papel importante nos primeiros anos de vida ao promover o crescimento e desenvolvimento da criança (Touchette, 2011 como referido por Mendonça, 2014).

A insuficiência e baixa qualidade do sono nas crianças tem vindo a constituir um problema de saúde pública (El-Sheikh e Buckhalt, s.d.), visto que 25 a $50 \%$ das crianças até aos cinco anos de idade apresentam dificuldades relacionadas com o sono (Mendonça, 2014). A manutenção de padrões de sono adequados ao estadio de desenvolvimento infantil é fundamental para a promoção de um crescimento e desenvolvimento saudável da criança. Deve-se atender não só à quantidade número de horas que a criança dorme por dia - como à qualidade do sono (Januário, 2012). Sabese que existe uma crescente relutância das crianças em adormecer, tendo consequentemente efeitos a longo prazo no desenvolvimento infantil com consequentes impactos negativos a nível comportamental, cognitivo e físico (El-Sheikh e Buckhal, s.d.). A análise de vários estudos permite recolher diversas informações referentes aos comportamentos que as crianças adotam antes de dormir. Há estudos que têm comprovado os efeitos nefastos dos meios eletrónicos no sono da criança, prin- 
cipalmente devido à luminosidade que pode alterar os níveis de melatonina, alterando o ciclo de sono e despertar, assim como o próprio conteúdo a que a criança assiste no tablet/telemóvel/computador que pode influenciar o seu comportamento antes de adormecer. Contudo, com os avanços tecnológicos, tem-se assistido a um mais fácil acesso e maior consumo de meios eletrónicos pelos mais jovens, sendo que muitas vezes recorrem aos mesmos antes de deitar, afetando assim, a qualidade de sono (Bathory e Tomopoulos, 2017). Por outro lado existem outros comportamentos que a criança manifesta antes de ir dormir. Destaca-se a ocorrência de comportamentos de choro e birras aquando da hora de adormecer. Estes comportamentos tornam-se muitas vezes uma preocupação para os pais de crianças em idade pré-escolar, pelo facto de não se sentirem capazes de mobilizar estratégias que os permitam prevenir e tranquilizar a criança (Cook et al., 2015).

Para um melhor controlo destes comportamentos torna-se fundamental estabelecer hábitos de sono saudáveis, nomeadamente um horário de sono estável, adormecer sozinho e eliminar meios eletrónicos (telemóveis, tablets, televisão, ...), promover uma adequada higiene do sono e quanto mais cedo ocorrer durante a infância, mais consolidados serão e melhor será a qualidade de sono da criança (Bonuck, Schwartz e Schechter, 2016).

Neste contexto, questionámo-nos "Como ajudam os enfermeiros os pais de crianças até aos cinco anos de idade a lidar com o comportamento dos filhos no momento de dormir?" Estabelecemos como objetivo geral: Conhecer as intervenções de enfermagem desenvolvidas pelos enfermeiros que visem 0 exercício adequado do papel parental no lidar com o comportamento dos filhos no momento de dormir. e objetivos específicos: Identificar as informações mais adequadas proporcionadas aos pais pelos enfermeiros que visem o exercício adequado do papel parental no lidar com o comportamento dos filhos no momento de dormir; Identificar o papel do enfermeiro na promoção do sono da criança; Identificar estratégias promotoras do sono na criança, adequadas à faixa etária dos 0 - 5 anos de idade e Identificar o papel do enfermeiro na promoção do papel parental no comportamento de sono da criança.

\section{MÉTODOS}

Revisão integrativa da literatura utilizando-se as palavras "parents"; "nurse"; "children’s sleep"; "Sleep habits"; "sleep"; "children" e "recommendation". Estabelecemos como critérios de inclusão que os estudos incluíssem, independente da metodologia de investigação utilizada, pais de crianças até aos cinco anos de idade e enfermeiros que assistem pais de crianças até aos cinco anos de idade. Tivéssemos acesso gratuito ao texto integral, que os estudos fossem publicados de $2012 \mathrm{a}$ Maio/2017, escritos em Português, Inglês ou Espanhol. Excluímos estudos que fizessem referência a crianças com perturbações do sono, pais de crianças portadoras de perturbações do sono, pais de crianças portadoras de patologia. Consultámos o portal Science Direct, acedendo às bases de dados: social science, nursing and heath professions, neuroscience, medicine and dentistry decision science. Identificaram-se 126 artigos, selecionaram-se 16 artigos e utilizaram-se quatro. Consultámos o portal B-on, acedendo às bases de dados: Psychology and Behavioral Sciences Collection; Medline (with full-text); CINAHL Plus (with full-text); MedicLatina, ERIC foram encontrados 569 artigos. Selecionamos 8 artigos e utilizámos três.

A terceira pesquisa teve como base o portal EBSCO, acedendo às bases de dados: Psychology and Behavioral Sciences Collection; Medline (with full-text); CINAHL Plus (with full-text); MedicLatina, ERIC. Encontraram-se 206 artigos, selecionaram-se 2 e utilizou-se um.

\section{RESULTADOS}

Do total dos oito estudos, três focavam as estratégias promotoras do sono da criança usadas pelos pais, dois analisaram os conhecimentos dos pais acerca do sono e dos comportamentos dos 


\section{PROMOÇÃO dO PAPEL PARENTAL NOS COMPORTAMENTOS DE SONO DE CRIANÇAS ATÉ AOS CINCO ANOS DE IDADE}

filhos no momento de dormir e cinco referiram o papel do enfermeiro e as estratégias usadas na promoção do papel parental no comportamento de sono da criança.

\section{Estratégias promotoras do sono da criança usadas pelos pais}

Mendonça (2014) no estudo que realizou com 123 pais de crianças dos 2 aos 6 anos para analisar as estratégias parentais relacionadas com o sono verificou que os pais para melhor promover o sono da criança adotam estratégias de conforto ativo, conforto passivo, relacionadas com 0 ambiente e rotinas e desadaptativas. Apurou que, as estratégias de conforto que os pais adotam, com maior frequência, são: " as festinhas", o dar a mão, visto que ajudam a sossegar e tranquilizar a criança. Relativamente às estratégias que englobam o conforto ativo como: "embalar a criança", tinham menor utilização ao passo que, relacionadas com o conforto passivo como: "deitar-se com a criança até que ela adormeça", eram as estratégias de moderado uso. Verificou ainda que existe uma elevada aplicação das estratégias relacionadas com as rotinas e 0 ambiente, sendo a mais frequente a estipulação da hora para a criança ir dormir. Por outro lado verificou um nível inferior de aplicação das estratégias desadaptativas, nomeadamente a utilização de televisão no momento de sossegar a criança.

Sinclair et al. (2016) para avaliar as preferências dos pais relativamente à realização da sesta dos filhos no infantário, inquiriu 750 pais de crianças entre os 3 e os 5 anos. Concluiu que $78,7 \%$ dos pais preferiam que os filhos não dormissem a sesta durante 0 dia, defendendo que se tornava contraproducente para o seu desenvolvimento, e que os restantes as defendiam, sendo que $10,1 \%$ defendiam sestas diárias.

Mileva-Seitz, Bakermans-Kranenburg, Battaini e Luijk (2016) analisaram a literatura existente sobre a partilha de cama durante a infância e avaliaram a prevalência desta prática a nível mundial bem como os benefícios/prejuízos que esta apresenta para a segurança e higiene de sono das crianças. Verificaram que a partilha da cama da criança com os pais pode ser utilizada de forma intencional ou devido a factores relacionados direta ou indiretamente com a criança. Observaram que esta prática poderá ocorrer durante toda noite ou apenas em parte, sendo que a primeira é realizada pelos pais que o fazem intencionalmente e a segunda devido a outros factores. A partilha difere de acordo com a cultura/etnia dos pais, contudo a sua utilização ao longo do desenvolvimento da criança apresenta tendência a diminuir. Verificaram existir diminuição desta prática, todavia algumas crianças não aceitam, chegando a fazer birras para que os pais não a abandonem. Existem vários motivos para a sua ocorrência: a criança apresentar-se irritada, estar doente, para facilitação da amamentação, segurança e conforto, despertares nocturnos, choro nocturno, pesadelos, birras das crianças no momento de deitar, diminuição da ansiedade e/ou devido a barreiras arquitectónicas (falta de outra divisão ou cama).

Os autores apuraram também que o Bed Sharing apresenta correlação positiva com sintomas depressivos nas mães e que consequentemente leva ao aumento da ansiedade e desespero da mãe.

Verificaram ainda que a prática do Bed Sharing, em bebés, pode provocar alterações na dinâmica do casal e alterações familiares.

\section{Conhecimentos dos pais acerca do sono e dos comportamentos dos filhos no momento de dor- mir}

McDowall, Galland e Campbel el Elder (2016) desenvolveram um estudo para compreender e analisar o conhecimento dos pais acerca do sono da criança e as atitudes promotoras do sono que estes apresentavam. Verificaram que $52 \%$ dos pais subestimava a necessidade dos filhos dormirem e $76 \%$ acreditavam que os filhos não têm um sono adequado. 0 estudo revelou ainda que os con- 
hecimentos dos pais relativamente ao sono das crianças era escasso e que os pais conseguem perceber se a duração do sono da criança é ou não adequada, contudo sentem dificuldade em identificar se o sono é ou não de qualidade. Uma outra dificuldade sentida pelos pais foi a identificação dos sinais e sintomas referentes ao sistema respiratório durante o sono (roncos, ressonar). Também observaram que os pais mostraram um número elevado de respostas erradas sobre conhecimentos acerca das consequências dos problemas no sono nocturno das crianças. Concluem que quanto maior o conhecimento dos pais acerca do sono dos filhos maior será a probabilidade de adoptarem estratégias adequadas, nomeadamente: adoção de um padrão de sono regular, deitar a criança cedo e sempre que possível à mesma hora, criar rotinas de sono, deixar que adormeçam sozinhas sem a sua presença, não permitir a TV ligada quando as crianças a têm no quarto.

Para averiguar os conhecimentos dos pais sobre o sono das crianças Bonuck, Schwartz e Schechter (2016) realizaram um estudo com 196 pais onde concluíram que mais de metade das crianças partilhava quarto com outros familiares (pais, irmãos) e mais de metade tinha ecrãs (televisão ou computador) no seu quarto. Uma pequena parte dos pais (10\%) acreditava que 0 filho sofria de distúrbios de sono, sendo que a maioria acreditava que o filho possuía hábitos de sono normais. Atestaram que cerca de metade dos pais afirmara não possuir conhecimentos suficientes para julgar a qualidade de sono do filho pelo que gostaria de adquirir mais informação sobre formas de melhorar a qualidade de sono. No entanto, mais de metade demonstrava conhecimentos sobre os efeitos da má qualidade de sono a longo prazo. Entre 30 a $80 \%$ dos pais responderam corretamente à secção que testava conhecimentos sobre higiene do sono, sendo que a maioria (81\%) tinha noção de que as crianças necessitam de estabelecer um horário de sono consistente desde cedo, contudo, alguns demonstravam-se menos elucidados sobre a necessidade de a criança manter uma rotina de sono (59\%), sobre sinais indicadores de que a criança está a ter um sono adequado (44 a $55 \%$ ) e dos efeitos da exposição a meios eletrónicos antes de dormir (58\%). Entre 50 a 60\% dos pais demonstravam-se cientes dos efeitos da privação de sono sobre o desenvolvimento e crescimento da criança a longo prazo. No que se refere à higiene do sono, a maior parte das famílias alegou ter um horário de sono regular (84\%), envolvendo uma atividade interativa (80\%).

\section{0 papel do enfermeiro e as estratégias usadas na promoção do papel parental no comporta- mento de sono da criança}

No estudo que Mendonça (2014) desenvolveu apurou fazer parte das competências do enfermeiro aconselhar e realizar educação parental de modo a haver controlo na qualidade do sono da criança, permitindo assim que os pais adoptem estratégias de acordo com as características temperamentais de cada criança, e a promoverem uma melhor qualidade do sono. Para os autores os enfermeiros devem ainda informar os pais sobre as implicações que algumas estratégias adoptadas podem ter na criança e suas consequências no sono.

Honaker e Meltzer (2015) desenvolveram um estudo para analisar a literatura existente sobre práticas relacionadas com 0 sono em contexto de cuidados de saúde primários, onde verificaram que para despistarem alterações no sono, apenas questionavam os pais acerca dos padrões de sono e sobre as suas preocupações acerca do sono da criança. Verificaram ainda que apenas $29 \%$ das enfermeiras pediatras, recebeu treino e formação acerca do sono e parte dessa foi realizada de forma autónoma em artigos científicos. De uma forma geral existe pouco ensino e formação nos cuidados primários pediátricos acerca do sono para profissionais de saúde 0 que dificulta a ação dos enfermeiros. Concluíram também que é essencial reforçar e realizar mais estudos acerca deste tema e reforçar a importância do sono na criança, que os enfermeiros devem questionar sempre que possível, a família ou a criança acerca do mesmo, assim como informar a família sobre a importância das rotinas e de outras intervenções relacionadas com o sono da criança. 
No estudo realizado por McDowall, Galland e Campbel el Elder, (2016) verificaram, que após os enfermeiros fornecerem um panfleto acerca do sono da criança aos pais, e estes o lerem, $83 \%$ dos pais conseguiram responder de forma correta a um elevado número de questões acerca do sono em idade pediátrica, aumentando também o número de pais que referiram que iriam planear de forma a melhorar os hábitos de sono dos seus filhos. Nesta perspectiva os autores verificaram que 0 ensino e 0 reforço dos ensinos realizados pelos enfermeiros aos pais das crianças, sobre as consequências dos problemas no sono nocturno das crianças são essenciais.

Em, 2016 Lopes realizou uma revisão sistemática da literatura com objetivo de mobilizar a prática baseada na evidência para os cuidados de enfermagem à criança e a sua família aquando de alterações de comportamentos de sono. Segundo a autora é o enfermeiro que tem como função minimizar as alterações do sono na criança, provocadas pelos diversos contextos, para tal deve usar o Modelo de parceria de cuidados de forma a estabelecer estratégias que possam ajudar os pais.

Segundo 0 estudo realizado por Oliviero e Brambilla, 2017 com objectivo de analisar o impacto das recomendações acerca do sono, as recomendações sobre o sono nas crianças desempenham um papel importante. Estas informações/recomendações devem ser colocadas sobre a forma de directrizes/ intervenções de forma a que os pais das crianças sejam informados sobre os comportamento de sono das suas crianças. Estes autores referem que a Academia Americana de Pediatria aconselha que crianças entre os 4 e os 12 meses durmam cerca de 16 horas, incluindo as sestas.

\section{DISCUSSÃO}

Relativamente à categoria: estratégias promotoras do sono da criança usadas pelos pais de crianças dos 0 aos 5 anos no momento de dormir 0 estudo de Mendonça (2014) mostrou que as estratégias relacionadas com o conforto, uma vez que estas conseguem tranquilizar e acalmar as crianças mais facilmente, e as rotinas e 0 ambiente são as mais utilizadas pelos pais para estabelecer rotinas no sono da criança, nomeadamente "as festinhas", o dar a mão e 0 estabelecimento da hora para a criança ir dormir. Também Cortina-Borja (2002) como referido por Mendonça (2014) referem que os pais das crianças ao longo do seu ciclo vital vão utilizando diversas estratégias. Para os autores quando a criança é pequena os pais tendem a utilizar estratégias direccionadas para 0 conforto e à medida que a criança se desenvolve utilizam estratégias mais direccionadas para a promoção da autonomia. Na perspectiva destes autores à medida que a criança se desenvolve, as estratégias de conforto ativo (por exemplo o embalar, o pegar na criança ao colo) tendem a reduzir, contudo as estratégias de conforto passivo (por exemplo deitar a criança) tendem a aumentar. Pais que estimulam a autonomia da criança e o seu auto-conforto, ajudam a promover melhor a qualidade do sono da sua criança e consequentemente, a diminuição dos despertares noturnos (Morrell \& Cortina-Borja, 2002 como referido Mendonça, 2014).

Owens (2004) e Sadeh (2009) como referido por Mendonça (2014) referem que os pais têm vindo a incentivar, cada vez mais, as suas crianças a dormirem nas suas próprias cama. Também Seitz, Kranenburd, Battaini e Luijk (2016) no seu estudo acerca do Bed Sharing referem que este é realizado por diversos motivos, apresentando um decréscimo do seu uso à medida que a criança se vai desenvolvendo, visto que é uma prática que apresenta benefícios e consequências para as crianças e suas famílias. Através deste estudo é possível concluir que os benefícios são inferiores aos prejuízos que advém desta prática para a criança e sua família, sendo que os principais prejuízos se encontram ligados às alterações familiares. Também Mindell, Sadeh, Kohyama e How (2010); Simard et al. (2008) e Owens (2004) como referidos por Mendonça (2014) defendem, respectivamente, associação entre 0 aparecimento de alterações/problemas no sono, 0 adormecimento da criança pelos pais, o alimentar a criança durante a noite e a partilha de cama. 
Sinclair et al. (2015) no estudo que desenvolveram verificaram que nos infantários é comum estabelecer uma rotina de sestas, independentemente do estadio de desenvolvimento da criança, 0 que nem sempre é favorável ao seu ritmo circadiano, sendo que alguns pais por vezes se queixam da interferência no sono nocturno.

Neste sentido, pode-se aferir a existência de uma discordância entre as preferências dos pais acerca das sestas diurnas e os protocolos dos infantários, pelo que é necessário ter especial atenção às práticas de sono em contexto de infantário e agir de forma a obter maior concordância tanto com as necessidades das crianças como as preferências dos pais. É neste sentido que, reconhecendo a Sociedade Portuguesa de Pediatria (2016) a importância da sesta nas crianças em idade pré-escolar, na aprendizagem recomenda que sejam proporcionadas condições adequadas (leito/colchão, ambiente calmo, escuro, com temperatura adequada, limitação de ruído e com vigilância) a todas as crianças em idade pré-escolar a fim de assegurar a qualidade do sono da sesta; que cada criança tenha um plano individual de sesta, acordado com a família e que a sesta seja promovida pela educadora de infância na presença de manifestações de privação de sono ou necessidade de sesta pela criança.

No que se refere à categoria: conhecimentos dos pais acerca do sono e dos comportamentos dos filhos no momento de dormir, na perspetiva de McDowall, Galland e Campbel el Elder (2016), apesar de os pais conseguirem perceber se a duração do sono é ou não adequada, revelam ainda, dificuldade em identificar se o sono é ou não de boa qualidade. Da mesma forma concluiu que, quanto mais vasto for o conhecimento dos pais acerca dos padrões de sono dos filhos, maior é a probabilidade de adotarem estratégias a fim de permitir uma melhor higiene de sono.

Por sua vez, Bonuck, Schwartz e Schechter (2015) concluíram que grande parte dos casos de pobre higiene de sono se encontravam relacionados com problemas de sono e que a presença frequente dos pais no quarto da criança aquando da hora de deitar se encontrava relacionado com variadas dificuldades de sono, nomeadamente sono insuficiente ao recomendado para a idade da criança. Do mesmo modo, muitas das rotinas que as crianças adotavam antes de deitar e os hábitos de utilização de meios eletrónicos não se encontravam associados a atitudes/crenças dos pais relativas a hábitos de sono. Outro aspeto foi o reconhecimento, pelos pais, de que a sua constante presença antes de adormecer afetava a qualidade do sono dos filhos, sendo que nestes casos, facilmente aceitavam e procuravam aconselhamento neste âmbito.

Assim, os resultados comprovam que os pais possuem uma compreensão ainda limitada sobre higiene de sono, pelo que há necessidade de reforçar ensinos.

Relativamente à categoria: o papel do enfermeiro e as estratégias usadas na promoção do papel parental no comportamento de sono da criança, na perspectiva de McDowall, Galland e Campbel el Elder (2016) é essencial o ensino e o reforço aos pais das crianças sobre as consequências dos problemas no sono nocturno das crianças. Assim como referido por Mendonça (2014) também Sadeh, Mindell, Luedtke e Wiegand (2009) recomendam que os enfermeiros informem os pais no sentido de incutirem nas suas crianças práticas de rotinas diárias na hora de adormecer uma vez que esta melhora a qualidade do sono.

Segundo a Ordem dos Enfermeiros (2001) como referido por Lopes (2016) durante a prestação de cuidados de enfermagem à criança 0 enfermeiro deve integrar no plano de cuidados, intervenções que visem a transformação de comportamentos e a promoção estilos de vida saudáveis conformáveis com a promoção da saúde. Assim é competência do enfermeiro transmitir à criança e sua família estratégias promotoras de um sono saudável, neste sentido, as estratégias devem ser adaptadas ao estadio de desenvolvimento da criança porém existem algumas estratégias universais a adotar como por exemplo: Deitar a criança, na sua cama, quando esta ainda se encontra acorda- 
da; Ensinar e incentivar a criança a dormir sozinha; A criança deve dormir na sua própria cama, no seu quarto, deve evitar-se que ela adormeça na cama dos pais; Estabelecer horários para a hora de dormir; Estabelecer um ritual no momento de dormir; Promover ambiente tranquilo e agradável, por exemplo deixar uma luz presença; Permitir o uso do objeto favorito e Diminuir o uso de equipamentos de estimulação (televisão, telemóveis, computadores, tablets) antes do momento de ir dormir (Brazelton \& Sparrow, 2009 como referido por Lopes, 2016). Pode- se concluir, que o enfermeiro apresenta um papel fundamental durante a prestação dos cuidados à criança e sua família.

\section{CONCLUSÃO}

0 sono, como necessidade humana fundamental, desempenha um papel importante no desenvolvimento da criança, podendo as alterações do sono levar a consequências nas diferentes áreas do desenvolvimento.

Tornou-se evidente que os pais adotam estratégias de promoção do sono da criança, de acordo com o seu nível de desenvolvimento. Contudo existem algumas disparidades na utilização de estratégias como o Bed Sharing e nas sestas em contexto de infantário.

Foi possível constatar que quanto maior for o leque de conhecimentos dos pais acerca da higiene e padrões de sono dos filhos, melhores são as estratégias que adotam uma vez que estas são adequadas às suas crianças e visam a promoção de comportamentos favorecedores do sono.

De uma forma geral, existe pouca formação sobre o sono dirigida aos enfermeiros o que dificulta a sua ação. Porém, faz parte das suas competências aconselhar e realizar educação parental de modo a haver controlo na qualidade do sono da criança, permitindo assim que os pais adoptem estratégias de acordo com as características temperamentais de cada criança, e a promoverem uma melhor qualidade do sono

0 enfermeiro deve recomendar estratégias como: deitar a criança, na sua própria cama, sendo que a criança deve estar acordada, devem estabelecer horários e um ritual para a hora de dormir, deve ensinar e incentivar os pais e a criança no sentido da criança dormir sozinha; explicar aos pais como podem promover um ambiente tranquilo e agradável, podem permitir 0 uso do objeto favorito, explicar aos pais as consequências que o uso de aparelhos eletrónicos como tablets, TV, videojogos, telemóveis podem trazer para o sono das suas crianças de modo a que os pais não permitam o seu uso no momento antes de ir dormir.

Nesta ótica, este estudo permitiu-nos concluir que embora os pais estejam, na maioria das vezes, sensíveis a desenvolver estratégias adequadas às necessidades de sono do filho, o enfermeiro deve, do mesmo modo, estar despertos para a necessidade de realizar ensinos de forma a capacitar os pais a agir melhor sobre o sono dos filhos, pelo que acreditamos que deverá ser feito um maior investimento na vertente dos ensinos e promoção de boas práticas antes do sono.

\section{REFERÊNCIAS BIBLIOGRÁFICAS}

Bathory, E., \& Tomopoulos, S. (2017). Sleep regulation, physiology and development, sleep duration and patterns, and sleep hygiene in infants, toddlers, and preschool-age children. Recuperado de https://www.ncbi.nlm.nih.gov/pubmed/28117135

Bonuck, K, A., Schwartz, B., \& Schechter, C. (2016). Sleep health literacy in head start families and staff: exploratory study of knowledge, motivation, and competencies to promote healthy sleep. Recuperado de http://www.sleephealthjournal.org/article/S2352- 7218(15)00190-4/pdf

Cook, F., Seymour, M., Giallo, R., Cann, W., Nicholson, M, J., Green, J., \& Hiscock, H. (2015). Comparison of methods for recruiting and engaging parents in online interventions: study protocol for the Cry Baby infant sleepand settling program. BMC Pediatrics doi 10.1186/s12887015-0502-9 
El-Sheikh, M., \& Buckhalt, A, J. (s.d.). Moving sleep and child development research forward: priorities and recommendations from the srcd-sponsored forum on sleep and child development.

Recuperado dhttp://onlinelibrary.wiley.com/doi/10.1111/mono.12142/epdf?r3_referer=wol\&tracking

_action=preview_click\&show_checkout $=1 \&$ purchase_referrer=www.google.pt\&purcha se_site_license=LICENSE_DENIED_NO_CUSTOMER

Honaker, M, S., \& Meltzer, J, L. (2015). Sleep in pediatric primary care: A review of the literature. Recuperado d http://www.sciencedirect.com/science/article/pii/S1087079215000167?via\%3Dihub

Januário, P. (2012). Hábitos de sono das crianças e compreensão infantil do sono e das estratégias facilitadoras do adormecimento: Estudo com crianças de oito anos e seus pais

(Dissertação de mestrado). Recuperado d e http://repositorio.ul.pt/bitstream/10451/8196/1/ulfpie043224_tm.pdf

Lopes, S. (2016). Estratégias para minimizar alterações do padrão de sono nas crianças dos 0 aos 3 anos (Tese de mestrado). Recuperado de http://repositorio.ipsantarem.pt/bitstream/10400.15/1396/1/trabalho\%20de\%20mestrado

$\% 20 \% 20$ Sandra\%20Lopes.pdf.

McDowall, S, P., Galland, C, B., Campbell, J, A., \& Elder, D. (2016). Parent knowledge of children's sleep: $\quad A \quad$ systematic review. Recuperado

de http://ac.els- cdn.com/S1087079216000058/1-s2.0-S1087079216000058main.pdf?_tid=7f3478ce- 5a62-11e7-b9f2-

00000aacb360\&acdnat=1498476654_df4504bf0a9e716710e739289afb7c4b

Mendonça, M. (2014). Determinantes individuais da qualidade do sono em crianças de idade préescolar (Tese de mestrado). Recuperado de http://repositorio.ul.pt/bitstream/10451/18229/1/ulfpie047210_tm.pdf

Mileva-Seitz, R, V., Bakermans-Kranenburg, J, M., Battaini , C., \& Luijk, M.C.P.M. (2016). Parentchild bed-sharing: The good, the bad, and the burden of evidence. Recuperado de http://ac.els-cdn.com/S1087079216000265/1-s2.0- S1087079216000265$\mathrm{m}$ a i n.p d f ? - t i d = f 00 e f a c $4-5$ a $62-11$ e $7-85$ d b 00000aab0f6c\&acdnat=1498476844_e7ab82e740a0870291de9fd73727c7a5

Oliviero, B., \& Brambilla, P. (2017). Impact of different recommendations on adequacy rate for sleep duration in children. Italian Journal of Pediatrics doi: 10.1186/s13052- 017-0329-0

Sinclair, D., Psych, M., Staton, S., Smith, S, S., Pattinson, L, C., Science, B. ... \& Thorpe,

K. (2016). What parents want: parent preference regarding sleep for their preschool child when attending early care and education. Recuperado de http://www.sciencedirect.com/science/article/pii/S2352721815001862

Sociedade Portuguesa de Pediatria. (2016). Recomendações sps-spp: prática da sesta da criança nas crechese infantários, públicos ou privados. Recuperado de http://www.spp.pt/UserFiles/file/Noticias_2017/VERSA0\%20PROFISSIONAIS\%20D E\%20SAUDE_RECOMENDACOES\%20SPSSPP\%20SESTA\%20NA\%20CRIANCA. pdf 
